\title{
PAIRED ACCELERATED FRAMES ${ }^{\dagger}$
}

\author{
ULRICH H. GERLACH \\ Department of Mathematics, Ohio State University, Columbus, OH 43210, USA
}

\begin{abstract}
The geometrical and quantum mechanical basis for Davies' and Unruh's acceleration temperature is traced to a type of quantum mechanical ("achronal") spin. Its existence and definition are based on pairs of causally disjoint accelerated frames. For bosons the expected spin vector of monochromatic particles is given by the "Planckian power" and the "r.m.s. thermal fluctuation" spectra. Under spacetime translation the spin direction precesses around that "Planckian" vector. By exhibiting the conserved achronal spin four-current, we extend the identification of achronal spin from single quanta to multiparticle systems. Total achronal spin conservation is also shown to hold, even in the presence of quadratic interactions.

In addition, the Lorentz invariance of the acceleration temperature is made explicit by the introduction of pairs of "spherical" Rindler frames.
\end{abstract}

Classical mechanics was the starting point by which Newton and Einstein brought gravitation into our grasp. Newton's particle trajectories carry the imprint of gravitational forces from which one can deduce the properties of gravitation and which hence led to Newton's equations for the gravitational potential. Einstein's worldlines, via geodesic deviation, carry the imprint of spacetime being curved, from which one can deduce gravitation as a manifestation of geometry, and which hence inevitably leads to Einstein's equations for the dynamics of the geometry.

The actual world is however quantum mechanical in nature, and the starting point for gravitation should reflect this fact. Thus the imprints of gravitation should not be carried by classical particle worldlines but, more fundamentally, by wave functions or operators of quantum mechanics instead.

\section{The Acceleration Temperature}

Nowadays, except for the energy $E=\hbar \omega$, it is difficult to point to a purely quantum mechanical formula which is simpler than that of Davies-Unruh (D-U) for the acceleration temperature $[1,2]$

$$
k T=\frac{\hbar}{c} \frac{g}{2 \pi}
$$

Can one therefore claim that in some way the simplicity of the D-U formula expresses a correspondingly fundamental aspect of nature? ["fundamental" meaning that, given the context of our present day knowledge, this aspect serves to organize the

$\dagger$ Based on a 'raporteur report' of the session "Quantum Radiation and Accelerated Frames" originally published in the Proc. of the Seventh Marcel Grossmann Meeting on General Relativity, Stanford, July 24-30, 1994, edited by R.T. Jantzen and M. Keiser (World Scientific, Singapore, 1996), 957-976. 
largest possible set of physical properties and phenomena into a readily identifiable existent]

The first thing one notices about the acceleration temperature is its ubiquity: the quantum dynamics of all relativistic wave equations that have been examined so far, be they for bosons $[1,2,3]$, fermions $[4,5]$, or for a nonlinear field [6], give rise to this temperature.

The second thing to notice is that it also occurs in gravitation: equate the acceleration $g$ to the surface gravity of a black hole event horizon,

$$
g=\frac{c^{4}}{4 M G} .
$$

With the gravitational constant $G$ introduced in this fashion, the acceleration temperature becomes the black hole temperature given by the Bekenstein-Hawking formula.

The third thing to notice is that the occurrence of the two constants of nature (the quantum of action $\hbar$, and the speed of light $c$ ) in the acceleration temperature somehow expresses an amalgamation of quantum mechanics with the causal ("light cone") structure of spacetime. Indeed, that causal structure is the geometrical corner stone of the $\mathrm{D}-\mathrm{U}$ formula. It is expressed by the pair of Rindler reference frames $(\xi>0)$,

$$
\begin{aligned}
& \left.t-t_{0}= \pm \xi \sinh g \tau\right\} \quad+: \text { "Rindler Sector I" } \\
& \left.z-z_{0}= \pm \xi \cosh g \tau\right\} \quad-\text { : "Rindler Sector II" }
\end{aligned}
$$

so that

$$
\left(z-z_{0}\right)^{2}-\left(t-t_{0}\right)^{2}=\xi^{2} .
$$

They accelerate linearly into opposite directions relative to the reference event $\left(t_{0}, x_{0}\right)$, and they each give rise to the same static spacetime geometry, which is expressed by

$$
d s^{2}=-\xi^{2} g^{2} d \tau^{2}+d \xi^{2}+d y^{2}+d x^{2} .
$$

This partitioning of spacetime into acceleration-induced pairs of adjacent but causally disjoint (i.e. "achronally" related) Rindler frames is expressed quantum mechanically by "achronal spin". This is done below.

\section{The Einstein Field Equations}

The nature of gravitation, i.e. the effect that it has on matter, as well as the manner in which matter determines gravitation, is expressed by the Einstein field equations. In their integral form they state that $[7,8]$

$$
\left(\begin{array}{c}
\text { "moment of } \\
\text { rotation" }
\end{array}\right)=8 \pi G \text { ("energy-momentum") }
$$


More precisely, the right hand side is the amount of energy and momentum per unit three-volume. The left hand side, on the other hand, is the moment of rotation associated with the six faces of this three-volume.

These equations depend on matter being characterized in terms of energy and momentum. To appreciate the significance of such a characterization, it is necessary to recall an important fact about dynamical variables in general, and energy and momentum in particular: They are of course attributes of single particles, and they are conserved for free particles in the absence of gravitation. However, equally or even more important for our purpose is the fact that they indicate and imply the nature of the coordinate frame.

If one chooses to characterize matter in terms of energy and momentum, (and this is what Einstein did when he developed his theory of gravitation) then one has implicitly committed himself to the use of instantaneous inertial ("free float") frames of reference at each event. In other words, energy-momentum is the physical expression of the translation invariance of free float frames. Thus the use of energy and momentum as dynamical variables is predicated on a picture of spacetime consisting of a locally translation invariant inertial frame (in geometry: "tangent space" with a basis) attached to each event. Once such a commitment has been made, geometry forces us to characterize these "tangent spaces" in terms of a metric, parallel transport, curvature, and so on, as one proceeds towards arriving at the Einstein's field equations.

To summarize, the Einstein field quations imply inertial frames of reference. The spacetime arena for the physics of matter is to be viewed in terms of "free float frames", one copy attached to each event.

\section{Inertial Frames vs Pairs of Accelerated frames}

The acceleration temperature implies a picture of spacetime which is in direct conflict with the picture implied by the Einstein field equations. The former is based on pairs of causally disjoint accelerated frames. The latter is based on instantaneous inertial frames of reference. Thus one has the peculiar state of affairs that the acceleration temperature implies pairs of accelerated frames, while the field equations imply inertial frames.

Even though both types of frames have a Cauchy hypersurface for initial value data of the relativistic equations of for matter, the difference in the classical as well as the quantum dynamics is irreconcilable. A pair of accelerated frames cannot be deformed into an inertial frame and vice versa. Ultimately one has to make a choice: pairs of accelerated frames implied by quantum mechanics, or inertial frames implied by the Einstein field equations.

\section{Lorentz Invariance}

If the acceleration temperature is to play a fundamental role in physics, then it must be in harmony with Lotentz invariance. This clearly does not seem to be the case: there is a preferred spatial direction along which the linear acceleration takes 
place. Moreover, the spacetime framework expressed by Eqs.(1.3)-(1.5) expresses another related deficiency: The pair of accelerated frames single out a reference event $\left(z-z_{0}=t-t_{0}=0\right)$ in the longitudinal spacetime plane, but the location of the frames in the transverse $(x-y)$ plane is left totally ambiguous.

However these deficiencies are removed by a frame which is characterized by accelerating spherical coordinates

$$
\begin{aligned}
t-t_{0} & =\xi \sinh g \tau \\
z-z_{0} & =\xi \cosh g \tau \cos \theta \\
y-y_{0} & =\xi \cosh g \tau \sin \theta \sin \phi \\
x-x_{0} & =\xi \cosh g \tau \sin \theta \cos \phi .
\end{aligned}
$$

The Minkowski metric relative to these coordinates is

$$
d s^{2}=-\xi^{2} g^{2} d \tau^{2}+d \xi^{2}+\xi^{2} \cosh ^{2} \tau\left(d \theta^{2}+\sin ^{2} \theta d \phi^{2}\right) .
$$

They coordinatize the Lorentz invariant histories of (fictitious) "bubbles" contracting and reexpanding around their reference event $\left(t_{0}, z_{0}, y_{0}, x_{0}\right)$. See Figure 1.

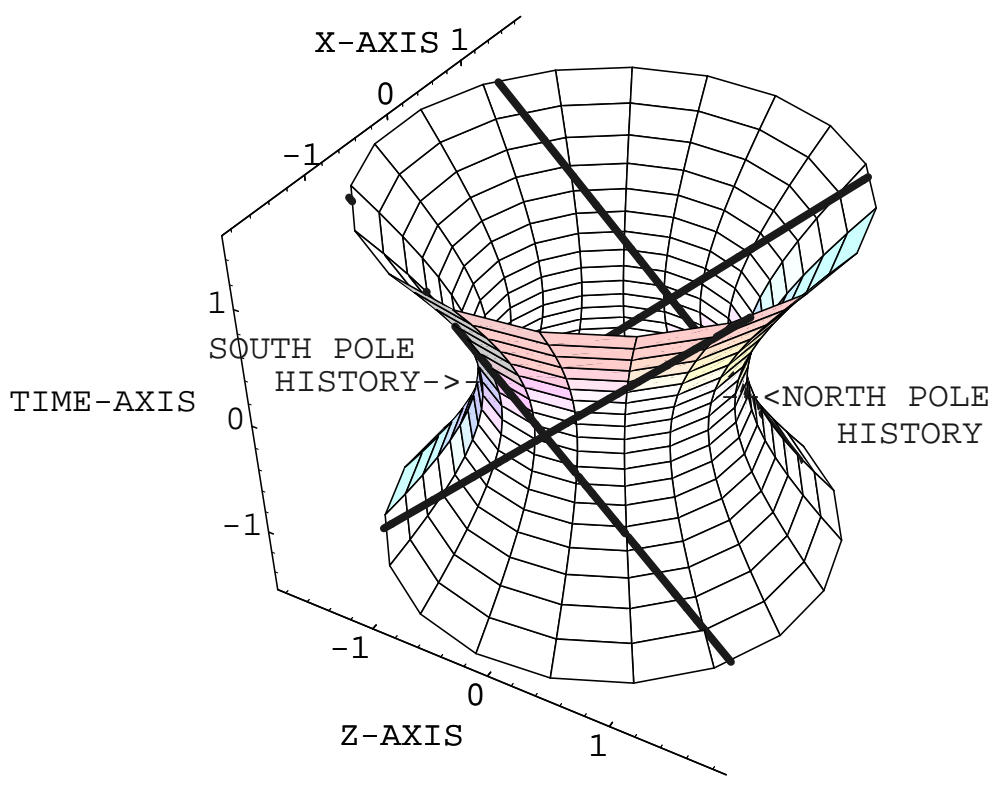

\section{FIGURE 1}

Lorentz invariant history of a contracting and reexpanding "bubble" partitioned by future and past event horizons (the heavy forty five 
degree lines) into a pair of causally disjoint (= "achronally" related) Rindler Sectors I and II. The y-dimension has been suppressed. The south and the north pole of the bubble trace out hyperbolic world lines in the respective Rindler Sectors I and II on the $\mathrm{x}=0$ plane. The history of the ergosphere consists of those events on the hyperboloid which lie between the event horizons and the two respective hyperbolas $\left(z-z_{0}\right)^{2}-\left(t-t_{0}\right)^{2}=\xi^{2} \cos ^{2} \theta, \pm\left|x-x_{0}\right|=\xi \sin \theta$, with vertices determined by Eq.(4.10).

The coordinates of this frame imply that each bubble has a hyperbolic history,

$$
\left(x-x_{0}\right)^{2}+\left(y-y_{0}\right)^{2}+\left(z-z_{0}\right)^{2}-\left(t-t_{0}\right)^{2}=\xi^{2},
$$

which is invariant under the actions of the Lorentz group $\mathrm{SO}(3,1)$ around the event $\left(t_{0}, z_{0}, y_{0}, x_{0}\right)$. This is a considerable improvement over the hyperbolic history

$$
\left(z-z_{0}\right)^{2}-\left(t-t_{0}\right)^{2}=\xi^{2}
$$

of a linearly accelerated frame, which lacks geometric isotropy, and which is ("boost") invariant under the actions of $S O(1,1)$ only. Furthermore, there is no ambiguity as to the spacetime location $\left(t_{0}, z_{0}, y_{0}, x_{0}\right)$ of the spherically symmetric frame.

However the key feature of this frame is quantum mechanical: each bubble is heated [9] to the acceleration temperature given by the $\mathrm{D}-\mathrm{U}$ formula

$$
k T=\frac{\hbar}{c} \frac{g}{2 \pi} \quad ;
$$

that is to say, a thermometer comoving with this bubble registers the acceleration temperature if the relativistic wavefield is in the Minkowski vacuum state.

Two geometric properties are responsible for this temperature: (a) the existence of a frame which is time invariant so that a relativistic wave system can come to thermal equilibrium at its acceleration temperature, and (b) the existence of past and future event horizons which partition the quantized relativistic wave field into a pair of causally disjoint subsystems.

\subsection{Spherical Rindler Frame}

With any Lorentz invariant "contracting and expanding bubble" frame coordinatized by Eq.(4.1) there is a concomitant unique frame with the required properties (a) and (b). This frame is coordinatized by the spherical Rindler coordinates [10],

$$
\begin{aligned}
& t-t_{0}=\xi \cos \theta \sinh \tau \\
& \left.z-z_{0}=\xi \cos \theta \cosh \tau\right\} \text { Rindler Sector I }: 0 \leq \theta<\frac{\pi}{2} \\
& \left.y-y_{0}=\xi \sin \theta \sin \phi\right\}\left\{\text { Rindler Sector II }: \frac{\pi}{2}<\theta \leq \pi\right. \text {, } \\
& x-x_{0}=\xi \sin \theta \cos \phi
\end{aligned}
$$


The past and future event horizons are

$$
\begin{aligned}
& z-z_{0}= \pm\left|t-t_{0}\right| \\
& y-y_{0}=\xi \sin \phi \\
& x-x_{0}=\xi \cos \phi, \quad 0<\xi<\infty, 0 \leq \phi<2 \pi .
\end{aligned}
$$

They partition the history of each concentric bubble into what for a linear Rindler frame corresponds to the pair of Rindler Sectors I and II in Eq.(1.3). See Figure 1. The north pole $(\theta=0)$ of each sphere has a hyperbolic world line in Rindler Sector I, while the south pole $(\theta=\pi)$ has a corresponding world line in Rindler Sector II. The metric on each of these Rindler sectors is

$$
d s^{2}=-\xi^{2} \cos ^{2} \theta d \tau^{2}+d \xi^{2}+\xi^{2}\left(d \theta^{2}+\sin ^{2} \theta d \phi^{2}\right),
$$

As required, the spherical Rindler frame is independent of time. The singularity in the metric

$$
\xi^{2} \cos ^{2} \theta=0,
$$

expresses the set of events where the past and the future event horizons intersect. These events are on the equatorial plane $z-z_{0}=0\left(\theta=\frac{\pi}{2}\right)$ of each contracting and reexpanding bubble at $t-t_{0}=0$, the moment of time symmetry as seen in the globally inertial frame $(t, z, y, x)$. These bifurcate equatorial events are the starting (or termination) events of the null ray histories of two swarms of null particles which travel into opposite directions parallel to the $z$-axis. The collection of these null ray histories make up the future and the past event horizons, Eq.(4.5). They form the boundaries of four mutually exclusive and jointly exhaustive coordinate neighborhoods on each hyperbolic "bubble" history, Eq.(4.2). They are the four Rindler Sectors ("wedges"): I and II, given by Eqs.(4.4), and the chronological future $(\mathrm{F})$ and past $(\mathrm{P})$, given by

$$
\begin{aligned}
& t-t_{0}=\xi \sinh \theta \cosh \tau \\
& \left.z-z_{0}=\xi \sinh \theta \sinh \tau\right\}\{\text { Rindler Sector F }: 0<\theta<\infty
\end{aligned}
$$

The metric on each of these two sectors is

$$
d s^{2}=\xi^{2} \sinh ^{2} \theta d \tau^{2}+d \xi^{2}+\xi^{2}\left(-d \theta^{2}+\cosh ^{2} \theta d \phi^{2}\right),
$$

\subsection{Rotating Spherical Rindler Frame and its Ergosphere}

The spherical Rindler coordinates associated with a set of contracting and reexpanding bubbles is not unique. It depends on the choice of a common axis of rotation around the chosen north pole and south pole of each bubble. This choice is equivalent to the choice of an equator. It determines the location of the north 
and south poles and hence the respective sectors I and II of the spherical Rindler frame. Put differently, there is one-to-one correspondence between a given set of bifurcate equatorial events and a spherical Rindler frame.

The existence of an axis of rotation permits the consideration of a spherical Rindler frame which rotates. This rotation is superimposed on the accelerative contraction and expansion of each bubble. If the common angular velocity is $\Omega$, then upon introducing the corotating angular coordinate

$$
\tilde{\phi}=\phi-\Omega \tau \text {, }
$$

the metric, Eq.(4.6), becomes

$$
d s^{2}=-\xi^{2}\left(\cos ^{2} \theta-\Omega^{2} \sin ^{2} \theta\right) d \tau^{2}+2 \Omega \xi^{2} \sin ^{2} \theta d \tilde{\phi} d \tau+d \xi^{2}+\xi^{2}\left(d \theta^{2}+\sin ^{2} \theta d \tilde{\phi}^{2}\right) .
$$

The ergosphere [11] is that region of space where the otherwise timelike Killing vec-

tor field $\frac{\partial}{\partial \tau}$ is spacelike. For a spherical Rindler frame with rotation the ergosphere is an equatorial belt. Its latitudinal thickness is given by

$$
-\Omega<\frac{\cos \theta}{\sin \theta}<\Omega
$$

independent of the radius of each sphere.

In spite of their geometrical difference, the $S O(1,1)$-based linear Rindler coordinate frames, Eq.(1.3), and the $S O(3,1)$-based spherical Rindler coordinate frames, Eq.(4.4), have a fundamental property in common: they both organize events into pairs of achronally related ("causally disjoint") spacetime regions I and II.

Such a partitioning into pairs introduces a qualitatively new feature into the quantum mechanics of a single particle, many particles ("quantum field theory"), as well as processes of interaction between them. This feature is achronal spin, which is developed below. The qualitative idea of achronal spin is easy to state. However its exact statement is in terms of the normal mode technology relative to Rindler sectors I and II. That technology is as-yet-undeveloped for spherical Rindler frames. Thus we shall use as a proxy the well-developed technology for pairs of linearly accelerated Rindler frames instead. This means that Lorentz invariance gets sacrificed in favor of simplicity. However the essential feature, achronal spin, is still present.

\section{Achronal Spin}

Consider the picture of spacetime which consists of pairs of oppositely accelerated reference frames, one pair attached to each event.

\subsection{Existence}


The physical consequences of such a geometrical picture is that the complete set of quantum mechanical observables of a particle governed by a linear relativistic wave equation has a qualitatively new member, "achronal spin".

Achronal spin is a quantum mechanical expression of acceleration-induced partitioning of spacetime into pairs of adjacent but causally disjoint (i.e. "achronally" related) Rindler sectors. Achronal spin is a conserved observable with a discrete spectrum, but, being a member of a complete set of observables, also indicates the pair of causally disconnected Rindler sectors at each reference event.

Assume for the sake of simplicity that these Rindler sectors result from a pair of frames accelerating linearly. Thus the two transverse dimensions become trivial and the spacetime arena becomes the two dimensional Lorentz plane. The boost invariance of the wave equation on this plane implies that the familiar boost energy is a conserved observable.

However there clearly has to be another observable: The relative phase and amplitude of the wave function in the two Rindler Sectors I and II can be changed at will without changing the boost energy and without destroying the fact that the wave function is a solution. In other words, the causally disjoint nature of the two Rindler domains I and II implies that a solution to the relativistic wave equation can be transformed into another solution by simply changing the relative phase and amplitude of the wave function defined on the two domains.

These changes form therefore an invariance group of the relativistic wave equation. This invariance group obviously commutes with Lorentz boosts. It follows that the generators of this group are observables which are also conserved. The two most important questions are: (1) What are the values of these observables? and (2) What is the "complete set of commuting observables" for the wave equation relative to the pair of Rindler frames I and II?

The answers to both questions follow from the representation and the Lie algebra of the invariance group. Consider a single scalar charge. The wave function on which the group acts has two components because the solution is defined on the two disconnected Rindler sectors, Eq.(1.3). Let this two component wave function be

$$
\psi(\tau, \xi)=\left[\begin{array}{c}
\psi_{\mathrm{I}}(\tau, \xi) \\
\psi_{\mathrm{II}}(\tau, \xi)
\end{array}\right]
$$

It satisfies the Klein Gordon equation in I and in II:

$$
\left[-\frac{1}{\xi^{2}} \frac{\partial^{2}}{\partial \tau^{2}}+\frac{1}{\xi} \frac{\partial}{\partial \xi} \xi \frac{\partial}{\partial \xi}-k^{2}\right]\left[\begin{array}{c}
\psi_{\mathrm{I}}(\tau, \xi) \\
\psi_{\mathrm{II}}(\tau, \xi)
\end{array}\right]=0
$$

Here $k^{2}=k_{x}^{2}+k_{y}^{2}+\frac{m^{2} c^{2}}{\hbar^{2}}$. The basic idea is illustrated by considering plane wave states

$$
e^{\mp i\left(t-t_{0}\right) k \cosh \theta+i\left(x-x_{0}\right) k \sinh \theta}
$$


of either positive (upper sign) or negative (lower sign) Minkowski frequency. These states, we recall, are parametrized by the "pseudo" angular parameter $\theta$ on the mass hyperboloid $\omega_{k}^{2}-k_{z}^{2}=k_{x}^{2}+k_{y}^{2}+\frac{m^{2}}{\hbar^{2}} \equiv k^{2}$,

$$
\begin{aligned}
\omega_{k} & =k \cosh \theta>0 \\
k_{z} & =k \sinh \theta
\end{aligned}
$$

In terms of the boost invariant normal modes $K_{i \omega}(k \xi) e^{-i \omega \tau}$, a plane wave state has an expansion consisting of two components, one for each of the two Rindler domains $I$ and $I I$, Eq.(1.3), on which it is defined. The result is [12]

$$
\begin{aligned}
\int_{-\infty}^{\infty}\left[\begin{array}{c}
e^{\pi \omega / 2} \\
e^{-\pi \omega / 2}
\end{array}\right] \frac{K_{i \omega}(k \xi)}{\pi} e^{-i \omega \tau} e^{i \omega \theta} d \omega & =\left[\begin{array}{c}
\left.e^{-i\left(t-t_{0}\right) k \cosh \theta+i\left(x-x_{0}\right) k \sinh \theta}\right|_{I} \\
\left.e^{-i\left(t-t_{0}\right) k \cosh \theta+i\left(x-x_{0}\right) k \sinh \theta}\right|_{I I}
\end{array}\right] \\
& =\left(\begin{array}{c}
\text { positive frequency } \\
\text { plane wave state }
\end{array}\right) \equiv \int_{-\infty}^{\infty} \psi_{\omega}^{+} d \omega
\end{aligned}
$$

and

$$
\begin{aligned}
\int_{-\infty}^{\infty}\left[\begin{array}{c}
e^{-\pi \omega / 2} \\
e^{\pi \omega / 2}
\end{array}\right] \frac{K_{i \omega}(k \xi)}{\pi} e^{-i \omega \tau} e^{i \omega \theta} d \omega & =\left[\begin{array}{c}
\left.e^{i\left(t-t_{0}\right) k \cosh \theta-i\left(x-x_{0}\right) k \sinh \theta}\right|_{I} \\
\left.e^{i\left(t-t_{0}\right) k \cosh \theta-i\left(x-x_{0}\right) k \sinh \theta}\right|_{I I}
\end{array}\right] \\
& =\left(\begin{array}{c}
\text { negative frequency } \\
\text { plane wave state }
\end{array}\right) \equiv \int_{-\infty}^{\infty} \psi_{\omega}^{-} d \omega
\end{aligned}
$$

Quantum mechanics demands that the action of the invariance group be such that the (Klein Gordon) inner product, Eq.(5.9) below, between quantum states remains invariant. Applied to each spectral component, this invariance determines the three one parameter subgroups

$$
\begin{gathered}
{\left[\begin{array}{cc}
\cosh \frac{\alpha}{2} & i \sinh \frac{\alpha}{2} \\
-i \sinh \frac{\alpha}{2} & \cosh \frac{\alpha}{2}
\end{array}\right] \equiv e^{i L_{1} \alpha},} \\
{\left[\begin{array}{cc}
\cosh \frac{\theta}{2} & \sinh \frac{\theta}{2} \\
\sinh \frac{\theta}{2} & \cosh \frac{\theta}{2}
\end{array}\right] \equiv e^{i L_{2} \theta}} \\
{\left[\begin{array}{cc}
e^{\frac{\phi}{2}} & 0 \\
0 & e^{\frac{\phi}{2}}
\end{array}\right] \equiv e^{i L_{3} \phi}}
\end{gathered}
$$

Here

$$
\vec{L}:\left\{L_{1}, L_{2}, L_{3}\right\}=\left\{\frac{1}{2}\left[\begin{array}{cc}
0 & 1 \\
-1 & 0
\end{array}\right], \frac{1}{2}\left[\begin{array}{cc}
0 & -i \\
-i & 0
\end{array}\right], \frac{1}{2}\left[\begin{array}{cc}
1 & 0 \\
0 & -1
\end{array}\right]\right\}
$$


are one half the modified Pauli spin matrices. They are the three achronal spin operators. They are conserved, and they generate the invariance group, which evidently is $S U(1,1)$. They satisfy the commutation relations

$$
\begin{aligned}
& {\left[L_{1}, L_{2}\right]=-i L_{3}} \\
& {\left[L_{2}, L_{3}\right]=i L_{1}} \\
& {\left[L_{3}, L_{1}\right]=i L_{2}}
\end{aligned}
$$

as well as

$$
\vec{L} \cdot \vec{L} \equiv L_{3}^{2}-L_{2}^{2}-L_{1}^{2}\left(=\frac{1}{2}\left(\frac{1}{2}+1\right)\right)
$$

One concludes that (1) a scalar charge has achronal spin $\frac{1}{2}$ and that (2) relative to a pair of Rindler frames in the two dimensional Lorentz plane the complete set of commuting observables for such a charge consists of the boost energy and the (third component of) achronal spin, $\left\{i \frac{\partial}{\partial \tau}, L_{3}\right\}$.

\subsection{Matrix Elements}

For any observable, including achronal spin, matrix elements are of particular physical interest: Among other things, they yield, of course, the expected spin orientation for a given quantum state.

This leads to a striking encounter between group theory and thermodynamics: The achronal spin expectation value determines a direction whose vertical component is "zero point energy" plus "Planckian power" while its horizontal component is the "r.m.s. thermal fluctuation". Nature is trying to tell us that quantum mechanical symmetries of spacetime manifest themselves thermodynamically through Boltzmann's maximum probability principle.

Matrix elements are based on a quantum mechanical inner product, which for a relativistic scalar charge is that of Klein Gordon [13],

$$
\begin{aligned}
\left\langle\psi, \psi^{\prime}\right\rangle & =\frac{i}{2} \int_{\infty}^{0}\left(\psi_{I I}^{*} \frac{\partial}{\partial \tau} \psi_{I I}^{\prime}-\frac{\partial}{\partial \tau} \psi_{I I}^{*} \psi_{I I}^{\prime}\right) \frac{d \xi}{\xi}+\frac{i}{2} \int_{0}^{\infty}\left(\psi_{I}^{*} \frac{\partial}{\partial \tau} \psi_{I}^{\prime}-\frac{\partial}{\partial \tau} \psi_{I}^{*} \psi_{I}\right) \frac{d \xi}{\xi} \\
& =\frac{i}{2} \int_{0}^{\infty}\left(\psi^{\dagger} \sigma_{3} \frac{\partial}{\partial \tau} \psi^{\prime}-\frac{\partial}{\partial \tau} \psi^{\dagger} \sigma_{3} \psi^{\prime}\right) \frac{d \xi}{\xi}
\end{aligned}
$$

Here $\sigma_{3}$ is the Pauli matrix

$$
\sigma_{3}=\left[\begin{array}{cc}
1 & 0 \\
0 & -1
\end{array}\right]
$$

Let us determine all the achronal spin matrix elements $\left\langle\psi_{\omega}^{+}, \vec{L} \psi_{\omega^{\prime}}^{+}\right\rangle,\left\langle\psi_{\omega}^{+}, \vec{L} \psi_{\omega^{\prime}}^{-}\right\rangle$, etc. for the pairs of positive and negative Minkowski frequency boost eigenstates 
$\psi_{\omega}^{+}$and $\psi_{\omega}^{-}$in Eq.(5.5). Introducing the "zero point energy" plus "Planckian power" spectrum

$$
\begin{aligned}
\frac{\cosh \pi \omega}{2 \sinh \pi \omega} & =\frac{1}{2}+\frac{1}{\exp 2 \pi \omega-1} \\
& \equiv \frac{1}{2}+N
\end{aligned}
$$

and the "r.m.s. thermal fluctuation" spectrum

$$
\frac{1}{2 \sinh \pi \omega}=\sqrt{N(N+1)}
$$

one obtains

$$
\begin{aligned}
& {\left[\begin{array}{cc}
\left\langle\psi_{\omega}^{+}, L_{1} \psi_{\omega^{\prime}}^{+}\right\rangle & \left\langle\psi_{\omega}^{+}, L_{1} \psi_{\omega^{\prime}}^{-}\right\rangle \\
\left\langle\psi_{\omega}^{-}, L_{1} \psi_{\omega^{\prime}}^{+}\right\rangle & \left\langle\psi_{\omega}^{-}, L_{1} \psi_{\omega^{\prime}}^{-}\right\rangle
\end{array}\right]=\left[\begin{array}{cc}
\sqrt{N(N+1)} & \frac{1}{2}+N \\
\frac{1}{2}+N & \sqrt{N(N+1)}
\end{array}\right] \delta\left(\omega-\omega^{\prime}\right)} \\
& {\left[\begin{array}{cc}
\left\langle\psi_{\omega}^{+}, L_{2} \psi_{\omega^{\prime}}^{+}\right\rangle & \left\langle\psi_{\omega}^{+}, L_{2} \psi_{\omega^{\prime}}^{-}\right\rangle \\
\left\langle\psi_{\omega}^{-}, L_{2} \psi_{\omega^{\prime}}^{+}\right\rangle & \left\langle\psi_{\omega}^{-}, L_{2} \psi_{\omega^{\prime}}^{-}\right\rangle
\end{array}\right]=} \\
& {\left[\begin{array}{cc}
0 & \frac{i}{2} \\
\frac{-i}{2} & 0
\end{array}\right] \delta\left(\omega-\omega^{\prime}\right)}
\end{aligned}
$$

and

$$
\left[\begin{array}{cc}
\left\langle\psi_{\omega}^{+}, L_{3} \psi_{\omega^{\prime}}^{+}\right\rangle & \left\langle\psi_{\omega}^{+}, L_{3} \psi_{\omega^{\prime}}^{-}\right\rangle \\
\left\langle\psi_{\omega}^{-}, L_{3} \psi_{\omega^{\prime}}^{+}\right\rangle & \left\langle\psi_{\omega}^{-}, L_{3} \psi_{\omega^{\prime}}^{-}\right\rangle
\end{array}\right]=\left[\begin{array}{cc}
\frac{1}{2}+N & \sqrt{N(N+1)} \\
\sqrt{N(N+1)} & \frac{1}{2}+N
\end{array}\right] \delta\left(\omega-\omega^{\prime}\right)
$$

while the inner products are simply

$$
\left[\begin{array}{cc}
\left\langle\psi_{\omega}^{+}, \psi_{\omega^{\prime}}^{+}\right\rangle & \left\langle\psi_{\omega}^{+}, \psi_{\omega^{\prime}}^{-}\right\rangle \\
\left\langle\psi_{\omega}^{-}, \psi_{\omega^{\prime}}^{+}\right\rangle & \left\langle\psi_{\omega}^{-}, \psi_{\omega^{\prime}}^{-}\right\rangle
\end{array}\right]=\quad\left[\begin{array}{cc}
1 & 0 \\
0 & -1
\end{array}\right] \delta\left(\omega-\omega^{\prime}\right)
$$

\subsection{Planckian Spectral Vector}

Relative to the quantum states ("spinors") $\psi_{\omega}^{+}$, as well as relative to $\psi_{\omega}^{-}$, the expectation value of the achronal spin operator $\mathbf{L}$ is

$$
\begin{aligned}
\left\langle\psi_{\omega}^{ \pm}, \mathbf{L} \psi_{\omega^{\prime}}^{ \pm}\right\rangle & \equiv \frac{1}{2} \mathbf{l}_{\omega}^{ \pm} \delta\left(\omega-\omega^{\prime}\right) \\
& =\left\{\left[\frac{1}{\exp \frac{\hbar \omega}{k T}-1}+\frac{1}{\left(\exp \frac{\hbar \omega}{k T}-1\right)^{2}}\right]^{\frac{1}{2}}, 0, \frac{1}{2}+\frac{1}{\exp \frac{\hbar \omega}{k T}-1}\right\} \delta\left(\omega-\omega^{\prime}\right) .
\end{aligned}
$$

These are the diagonal matrix elements. The vector $\mathbf{l}_{\omega}^{+}$, as well as $\mathbf{l}_{\omega}^{-}$, formed from these expectation values is a real three-dimensional vector, which we shall call the "Planckian spectral vector". This vector is unique. It is the same for all plane wave states, and its components are two of Nature's most ubiquitous spectra: the "zero point energy" plus "Planckian thermal power" spectrum for the third component and the "r.m.s. thermal fluctuation" spectrum for the first component. The Planckian spectral vector has a dynamical significance which is described in the next subsection. 
The off-diagonal elements are complex. Their real and imaginary parts

$$
\begin{aligned}
\left\langle\psi_{\omega}^{\mp}, \mathbf{L} \psi_{\omega^{\prime}}^{ \pm}\right\rangle & =\operatorname{Re}\left\langle\psi_{\omega}^{\mp}, \mathbf{L} \psi_{\omega^{\prime}}^{ \pm}\right\rangle+i \operatorname{Im}\left\langle\psi_{\omega}^{\mp}, \mathbf{L} \psi_{\omega^{\prime}}^{ \pm}\right\rangle \\
& \equiv\left(\frac{1}{2} \mathbf{m}_{\omega}^{ \pm}+i \frac{1}{2} \mathbf{n}_{\omega}^{ \pm}\right) \quad \delta\left(\omega-\omega^{\prime}\right)
\end{aligned}
$$

yield two addtional real three-dimensional vectors,

$$
\begin{aligned}
\mathbf{m}_{\omega}^{ \pm} & =2\left\{\frac{1}{2}+\frac{1}{\exp \frac{\hbar \omega}{k T}-1}, 0,\left[\frac{1}{\exp \frac{\hbar \omega}{k T}-1}+\frac{1}{\left(\exp \frac{\hbar \omega}{k T}-1\right)^{2}}\right]^{\frac{1}{2}}\right\} \\
\mathbf{n}_{\omega}^{ \pm} & = \pm\{0,1,0\} .
\end{aligned}
$$

Together the vectors $\mathbf{l}_{\omega}^{+}, \mathbf{m}_{\omega}^{+}$, and $\mathbf{n}_{\omega}^{+}$form a triad, which is "Lorentz" orthonormal in the sense that

$$
\begin{aligned}
\mathbf{l}_{\omega}^{+} \cdot \mathbf{l}_{\omega}^{+} & \equiv\left(l_{\omega 3}\right)^{2}-\left(l_{\omega 2}\right)^{2}-\left(l_{\omega 1}\right)^{2} \\
& =1 \\
\mathbf{m}_{\omega}^{+} \cdot \mathbf{m}_{\omega}^{+} & =\mathbf{n}_{\omega}^{+} \cdot \mathbf{n}_{\omega}^{+}=-1 \\
\mathbf{l}_{\omega}^{+} \cdot \mathbf{m}_{\omega}^{+} & =\mathbf{l}_{\omega}^{+} \cdot \mathbf{n}_{\omega}^{+}=\mathbf{l}_{\omega}^{+} \cdot \mathbf{n}_{\omega}^{+}=0
\end{aligned}
$$

The directions of the vectors making up this triad are unique. Like the Planckian spectral vector, they are the same for all plane wave states and their components are fixed by Nature.

\subsection{Precession under Spacetime Translation}

We now ask and answer two questions: (1) How does achronal spin change in the paired Rindler frame attached to a given fixed event? (2) How does achronal spin change under spacetime tranlations from the paired frame at one event to the paired frame at another?

The quick answer to the first question is that it does not change at all; it remains fixed under boost time evolution. The answer to the second question is that it precesses around the direction of the Planckian spectral vector.

Let us see how the Klein-Gordon wave equation furnishes the answer to these two questions. Consider the expectation value taken with respect to an arbitrary Klein-Gordon state,

$$
\begin{aligned}
\psi & =\int_{-\infty}^{\infty}\left[\begin{array}{c}
a_{\omega} \\
b_{\omega}^{*}
\end{array}\right] \frac{K_{i \omega}(k \xi)}{\pi} e^{-i \omega \tau} d \omega \\
& \equiv \int_{-\infty}^{\infty} \psi_{\omega} d \omega .
\end{aligned}
$$


More precisely, take the expectation values of $\mathbf{L}:\left(L_{1}, L_{2}, L_{3}\right)$ with respect to the spectral components $\psi_{\omega}$. Being based on the standard quantum mechanical (KleinGordon) inner product, Eq.(5.9)

$$
\left\langle\psi_{\omega}, \psi_{\omega^{\prime}}\right\rangle=\left(a_{\omega}^{*} a_{\omega}-b_{\omega} b_{\omega}^{*}\right) \frac{\delta\left(\omega-\omega^{\prime}\right)}{2 \sinh \pi \omega}
$$

these expectation values are (by inspection of Eq.(5.7))

$$
\begin{aligned}
\left\langle\psi_{\omega}, L_{1} \psi_{\omega^{\prime}}\right\rangle & =\frac{1}{2}\left(a_{\omega} b_{\omega}+a_{\omega}^{*} b_{\omega}^{*}\right) \frac{\delta\left(\omega-\omega^{\prime}\right)}{2 \sinh \pi \omega} \\
\left\langle\psi_{\omega}, L_{2} \psi_{\omega^{\prime}}\right\rangle & =-\frac{i}{2}\left(a_{\omega} b_{\omega}-a_{\omega}^{*} b_{\omega}^{*}\right) \frac{\delta\left(\omega-\omega^{\prime}\right)}{2 \sinh \pi \omega} \\
\left\langle\psi_{\omega}, L_{3} \psi_{\omega^{\prime}}\right\rangle & =\frac{1}{2}\left(a_{\omega}^{*} a_{\omega}+b_{\omega} b_{\omega}^{*}\right) \frac{\delta\left(\omega-\omega^{\prime}\right)}{2 \sinh \pi \omega} .
\end{aligned}
$$

The time (i.e $\tau$, the "boost" time) independence of these expressions imply the first result: In any given paired accelerated frame achronal spin $\mathbf{L}:\left(L_{1}, L_{2}, L_{3}\right)$ is a (set of) constant(s) of motion. Although not simultaneously measurable, they are nevertheless fixed and conserved. This answers the first question posed at the beginning of this section.

What about the second question, change of achronal spin under spacetime translation? There is a definite answer, "precession". It is illustrated by considering what in a global inertial frame is an arbitrary superposition of (i) a positive Minkowski frequency ("particle", (5.5a)) plane wave state and (ii) another plane wave state of negative Minkowski frequency ("antiparticle", (5.5b)). Let the amplitudes of these states be $\tilde{a}$ and $\tilde{b}^{*}$ respectively [15]:

$$
\begin{aligned}
\psi & =\tilde{a} \int_{-\infty}^{\infty} e^{i \omega \theta_{1}}\left[\begin{array}{c}
e^{\pi \omega / 2} \\
e^{-\pi \omega / 2}
\end{array}\right] \frac{K_{i \omega}(k \xi)}{\pi} e^{-i \omega \tau} d \omega+\tilde{b}^{*} \int_{-\infty}^{\infty} e^{i \omega \theta_{2}}\left[\begin{array}{c}
e^{-\pi \omega / 2} \\
e^{\pi \omega / 2}
\end{array}\right] \frac{K_{i \omega}(k \xi)}{\pi} e^{-i \omega \tau} d \omega \\
& \equiv \int_{-\infty}^{\infty}\left\{\tilde{a} \psi_{\omega}^{+}+\tilde{b}^{*} \psi_{\omega}^{-}\right\} d \omega \\
& \equiv \int_{-\infty}^{\infty} \tilde{\psi}_{\omega} d \omega
\end{aligned}
$$

What is the spectral achronal spin direction when the charge is in this state, and how does this direction change under spacetime translations? Before such a translation the expected spin direction is

$$
\begin{aligned}
\frac{\left\langle\tilde{\psi}_{\omega}, \mathbf{L} \tilde{\psi}_{\omega^{\prime}}\right\rangle}{\left\langle\tilde{\psi}_{\omega}, \tilde{\psi}_{\omega^{\prime}}\right\rangle}= & \frac{|\tilde{a}|^{2}+|\tilde{b}|^{2}}{|\tilde{a}|^{2}-|\tilde{b}|^{2}} \frac{\mathbf{l}_{\omega}}{2} \\
& +\frac{2|\tilde{a}||\tilde{b}|}{|\tilde{a}|^{2}-|\tilde{b}|^{2}}\left[\frac{\mathbf{m}_{\omega}}{2} \cos \delta+\frac{\mathbf{n}_{\omega}}{2} \sin \delta\right] .
\end{aligned}
$$


Here $\delta=\delta_{1}+\delta_{2}$ is the sum of the phase angles of the two amplitudes

$$
\begin{aligned}
& \tilde{a}=|\tilde{a}| e^{i \delta_{1}} \\
& \tilde{b}=|\tilde{b}| e^{i \delta_{2}}
\end{aligned}
$$

Now subject the plane wave state in Eqs.(5.5) to a translation. This action alters the basis spinors $\left[\begin{array}{ll}e^{\pi \omega / 2} & e^{-\pi \omega / 2}\end{array}\right]^{\text {transpose }}$ in Eq.(5.5a) by a common $\omega$-independent phase factor. When introduced into Eq.(5.18) (in order to subject $\psi$ to the same translation) this phase factor appears as a multiplier of the coefficient $\tilde{a}$ under the integral. In other words, a translation is represented by multiplying the amplitude $\tilde{a}$ by a common phase factor. Similarly, $\tilde{b}^{*}$ also gets multiplied by a (different) phase factor:

$$
\begin{aligned}
\tilde{a} & \rightarrow \tilde{a} e^{i \gamma_{1}} \\
\tilde{b} & \rightarrow \tilde{b} e^{i \gamma_{2}}
\end{aligned}
$$

The effect of these spacetime translation-induced phase changes is to rotate the expected achronal spin around the Planckian spectral direction

$$
\mathbf{l}_{\omega}=\left\{\left[\frac{1}{\exp \frac{\hbar \omega}{k T}-1}+\frac{1}{\left(\exp \frac{\hbar \omega}{k T}-1\right)^{2}}\right]^{\frac{1}{2}}, 0, \frac{1}{2}+\frac{1}{\exp \frac{\hbar \omega}{k T}-1}\right\}
$$

by the angle $\gamma=\gamma_{1}+\gamma_{2}$ :

$$
\begin{aligned}
\frac{\left\langle\tilde{\psi}_{\omega}, \mathbf{L} \tilde{\psi}_{\omega^{\prime}}\right\rangle}{\left\langle\tilde{\psi}_{\omega}, \tilde{\psi}_{\omega^{\prime}}\right\rangle} \rightarrow & \frac{|\tilde{a}|^{2}+|\tilde{b}|^{2}}{|\tilde{a}|^{2}-|\tilde{b}|^{2}} \frac{\mathbf{l}_{\omega}}{2} \\
& +\frac{2|\tilde{a}||\tilde{b}|}{|\tilde{a}|^{2}-|\tilde{b}|^{2}}\left[\frac{\mathbf{m}_{\omega}}{2} \cos (\delta+\gamma)+\frac{\mathbf{n}_{\omega}}{2} \sin (\delta+\gamma)\right] .
\end{aligned}
$$

The hyperbolic angle of inclination of the spin axis from the Planckian vector is given by

$$
\tanh \alpha=\frac{2|\tilde{a}||\tilde{b}|}{|\tilde{a}|^{2}+|\tilde{b}|^{2}}
$$

The angular amount by which the spin axis precesses around the Planckian vector is proportional to the spacetime displacement $(\triangle t, \triangle x)$. Indeed, the plane wave states Eq.(5.5) imply that this precession angle is

$$
\gamma \equiv \gamma_{1}+\gamma_{2}=\triangle t\left(\cosh \theta_{1}+\cosh \theta_{2}\right) k-\triangle x\left(\sinh \theta_{1}+\sinh \theta_{2}\right) k
$$




\section{Paired Accelerated Frames: Interacting Multiparticle Systems}

Gravitation manifests itself by the imprints it leaves on classical or, more fundamentally, on quantum mechanical processes. The imprints on the orbits and world lines of particles and even on wave fields are well-known and appreciated. Such a harmonious and universally accepted assessment does not, however, prevail for the imprints that gravitation leaves on individual quanta. The problem is that, in the absence of appropriate symmetries, gravitation introduces an ambiguity as to the distinction between positive and negative frequency field oscillators. As a consequence, there is an ambiguity as to the very definition of what is meant by a particle, i.e. a carrier of energy and momentum. To tackle this conundrum, the viewpoint has been advocated that a particle be identified by the fact that it brings about a change in the state of a detecting apparatus which follows some prespecified world line.

In practical terms this means that the onus of the ambiguity of the negativepositive frequency distinction has been shifted to the arbitrariness of the detector world line.

This state of affairs is somewhat reminiscent of the problem Euclidean geometers faced before Riemannian geometry: "Given a straight line ("geodesic"), through a close-by point, draw another straight line parallel line parallel to the first." In the absence of appropriate symmetries of the manifold, a pair of straight lines can not be identified unambiguously as having positive or negative relative slope. To deal with this problem Riemann's qualitatively different viewpoint had to be taken.

\subsection{Binary Frame Picture of Spacetime}

One is led therefore to consider that the quantum mechanical positive-negative frequency ambiguity introduced by gravitation requires an analogous change in viewpoint, i.e. in the way we picture spacetime. In other words, this ambiguity is evidence of a to-be-identified geometrical structure which is a quantum mechanical carrier of the imprints of gravitation.

What is the framework which would permit the accomodation of such imprints? The ubiquity of the acceleration temperature, Eq.(1.1), demands that this framework consist of viewing spacetime in a qualitatively different way: Instead of assigning to each event a locally inertial ("free float") reference frame (i.e. a tangent space with a basis), spacetime is to be pictured as a union of pairs of accelerated reference frames, which are achronally related and whose future and past event horizons intersect at the base event to which a given pair is attached.

Let us call this demand the "binary frame hypothesis". It is expressed quantum mechanically by the fact that "achronal spin exists". In other words, "binary frames" play a central role in physics only to the extent that achronal spin does. The binary frame picture of spacetime and the existence of achronal spin go hand in hand. They cannot be separated.

The binary aspect of a pair of accelerated frames becomes particularly significant when interactions are present. In other words, it is of importance that the 
domain of the two relativistic quantum systems include both Rindler sectors. Much physical insight has been gained from the path breaking considerations involving a multiparticle quantum ("quantized field") system interacting with an "accelerated detector" confined to a single Rindler frame [2,16-20]. However, physics demands that an "accelerated detector" not discriminate against one of the Rindler components of the multiparticle quantum system. The "accelerated detector" should in fact consist of a pair of detectors, one in each Rindler sector. At a minimum one can always recover the well known single detector results by tracing out the quantum states of the other detector.

Using a pair of detectors is not a luxury. Instead, it becomes a necessity if one wishes to characterize the results of the interaction in terms of the conservation of total achronal spin for the combined field-plus- "detector pair" quantum system. Furthermore, using a pair of detectors becomes unavoidable if one considers translations in spacetime.

The definition and conservation of achronal spin for the two interacting quantum systems is illustrated by the following simple and exactly soluble example.

\subsection{Two Interacting Quantum Systems}

Consider two infinite quantum systems. The first, the "field system", has Klein Gordon quanta of rest mass $m$, while the second one, the "detector pair system" has different quanta of rest mass $\mu$. The domain of both systems extends over all of spacetime, in particular over a given binary frame consisting of the two Rindler frames, Eq.(1.3). Collisions between the $m$ and the $\mu$ quanta are brought about by an interaction whose strength is proportional to the coupling constant $e^{2}$. The wave equation for the two systems are

$$
\begin{aligned}
\left(\square-m^{2}\right) \psi & =e^{2} \phi \\
\left(\square-\mu^{2}\right) \phi & =e^{2} \psi
\end{aligned}
$$

Notice the simplicity of our assumption. It is expressed by the interaction Hamiltonian

$$
\begin{aligned}
H_{i n t} & =e^{2} \int_{0}^{\infty}\left(\psi^{\dagger} \sigma_{3} \phi+\phi^{\dagger} \sigma_{3} \psi\right) \xi d \xi \\
& \equiv e^{2} \int_{0}^{\infty}\left\{\left[\psi_{I}^{*} \psi_{I I}^{*}\right] \sigma_{3}\left[\begin{array}{c}
\phi_{I} \\
\phi_{I I}
\end{array}\right]+\left[\begin{array}{ll}
\phi_{I}^{*} & \phi_{I I}^{*}
\end{array}\right] \sigma_{3}\left[\begin{array}{c}
\psi_{I} \\
\psi_{I I}
\end{array}\right]\right\} \xi d \xi
\end{aligned}
$$

which is quadratic. The two wave equations can be decoupled by means of an orthogonal transformation, but we shall not have to do this.

Observable quantities and their dynamical evolution derive their importance from their ability to carry the imprints of gravitation. The two key questions are therefore

(1) What are the observables?

(2)What is their dynamical evolution as governed by the coupled equations of motion, Eq.(6.1)? 
It is essential to answer these questions first in the absence of gravitation. At another time, when gravitation is present the answer is different. This difference is the imprint left by gravity.

\subsection{Achronal Spin and the Achronal Spin Four-Current}

To identify the appropriate observables, we incorporate the binary nature of the pair of Rindler frames into a differential conservation law. Considerations based on the inner product, Eq.(5.9), and the expectation values of $\vec{L}$ imply that one introduce the achronal spin four-current

$$
\begin{aligned}
\left(\overrightarrow{J^{\psi}}\right)_{\mu} & =i\left\{\left[\begin{array}{ll}
\psi_{I}^{*} & \psi_{I I}^{*}
\end{array}\right] \sigma_{3} \vec{L}\left[\begin{array}{c}
\nabla_{\mu} \psi_{I} \\
\nabla_{\mu} \psi_{I I}
\end{array}\right]-\left[\begin{array}{ll}
\nabla_{\mu} \psi_{I}^{*} & \nabla_{\mu} \psi_{I I}^{*}
\end{array}\right] \sigma_{3} \vec{L}\left[\begin{array}{c}
\psi_{I} \\
\psi_{I I}
\end{array}\right]\right\} \\
& \equiv i\left\{\psi^{\dagger} \sigma_{3} \vec{L} \nabla_{\mu} \psi-\nabla_{\mu} \psi^{\dagger} \sigma_{3} \vec{L} \psi\right\}
\end{aligned}
$$

In the absence of interaction $\left(e^{2}=0\right)$ its divergence vanishes. However, if interactions are present, then with the help of Eqs.(6.1) the divergence is

$$
\nabla^{\mu}\left(\overrightarrow{J^{\psi}}\right)_{\mu}=e^{2} i\left(\psi^{\dagger} \sigma_{3} \vec{L} \phi-\phi^{\dagger} \sigma_{3} \vec{L} \psi\right)
$$

The fourth component of the four-current is a density, that of achronal spin, as we presently shall see. The integral of this density is the total amount of (vectorial) achronal spin relative to a given binary frame. Using the inner product notation of Eq.(5.9) this vector is

$$
\begin{aligned}
\overrightarrow{J^{\psi}} & =i \int_{0}^{\infty}\left(\psi^{\dagger} \sigma_{3} \vec{L} \frac{\partial}{\partial \tau} \psi-\frac{\partial}{\partial \tau} \psi^{\dagger} \sigma_{3} \vec{L} \psi\right) \frac{d \xi}{\xi} \\
& \equiv 2\langle\psi, \vec{L} \psi\rangle .
\end{aligned}
$$

Within the framework of quantum mechanics both $\psi$ and $\overrightarrow{J^{\psi}}$ are operators. What compells us to identify the components $J_{i}^{\psi}(i=1,2,3)$, of $\overrightarrow{J^{\psi}}$ as the achronal spin components of the $\psi$ quantum system? Answer: The quantum mechanical equal time ( $\tau$ ") commutation relations

$$
\begin{aligned}
& {\left[\dot{\psi}_{I}^{*}(\xi, \tau), \psi_{I}\left(\xi^{\prime}, \tau\right)\right] }=-i \xi \delta\left(\xi-\xi^{\prime}\right) \\
& {\left[\dot{\psi}_{I}^{*}(\xi, \tau), \psi_{I I}\left(\xi^{\prime}, \tau\right)\right] }=0 \\
& \vdots \\
& \text { etc. }
\end{aligned}
$$

of the $\psi$ system. They imply that

$$
\begin{aligned}
& {\left[J_{1}^{\psi}, J_{2}^{\psi}\right]=-i J_{3}^{\psi}} \\
& {\left[J_{2}^{\psi}, J_{3}^{\psi}\right]=i J_{1}^{\psi}} \\
& {\left[J_{3}^{\psi}, J_{1}^{\psi}\right]=i J_{2}^{\psi}}
\end{aligned}
$$


These commutation relations of the quantum operators $J_{i}^{\psi}$ are those that characterize the $J_{i}^{\psi}$ as the achronal spin components, the generators of the achronal symmetry group $S U(1,1)$.

\subsection{Conservation of Achronal Spin}

The $\phi$ quantum system has its corresponding achronal spin $\overrightarrow{J^{\phi}}$ whose components also satisfy the $S(1,1)$ commutation relations

$$
\begin{aligned}
& {\left[J_{1}^{\phi}, J_{2}^{\phi}\right]=-i J_{3}^{\phi}} \\
& {\left[J_{2}^{\phi}, J_{3}^{\phi}\right]=i J_{1}^{\phi}} \\
& {\left[J_{3}^{\phi}, J_{1}^{\phi}\right]=i J_{2}^{\phi}}
\end{aligned}
$$

The corresponding achronal spin four-current for the $\phi$ system is defined in the same way as in Eq.(6.3), and its divergence is

$$
\nabla^{\mu}\left(\overrightarrow{J^{\phi}}\right)_{\mu}=e^{2} i\left(\phi^{\dagger} \sigma_{3} \vec{L} \psi-\psi^{\dagger} \sigma_{3} \vec{L} \phi\right)
$$

which does not vanish either. However, the divergence of total achronal spin current, $\left(\overrightarrow{J^{\psi}}\right)_{\mu}+\left(\overrightarrow{J^{\phi}}\right)_{\mu}$ does vanish,

$$
\nabla^{\mu}\left[\left(\overrightarrow{J^{\psi}}\right)_{\mu}+\left(\overrightarrow{J^{\phi}}\right)_{\mu}\right]=0
$$

Thus one sees that in the presence of interaction $\left(e^{2} \neq 0\right)$ none of the individual achronal spins, $\overrightarrow{J^{\psi}}$ and $\overrightarrow{J^{\phi}}$, are conserved. However, the achronal spin of the total system,

$$
\overrightarrow{J^{\text {total }}}=\overrightarrow{J^{\psi}}+\overrightarrow{J^{\phi}}
$$

is conserved throughout the interaction process.

\subsection{Conserved Total Boost Energy and Total Charge}

In addition to the total achronal spin components, the binary frame observables of the relativistic system include the total "boost Hamiltonian". Using the the inner product notation of Eq.(5.9), this Hamiltonian is

$$
H^{\text {total }}=2\left\langle\psi, i \frac{\partial}{\partial \tau} \psi\right\rangle+2\left\langle\phi, i \frac{\partial}{\partial \tau} \phi\right\rangle
$$

for the two interacting systems $\psi$ and $\phi$. With the help of the wave Eqs.(6.1) and an integration this becomes the familiar sum

$$
H^{\text {total }}=H^{\psi}+H^{\phi}+H_{\text {int }}
$$


of the $\psi$-system Hamiltonian

$$
H^{\psi}=\int_{0}^{\infty}\left\{\frac{1}{\xi^{2}} \frac{\partial \psi^{\dagger}}{\partial \tau} \sigma_{3} \frac{\partial \psi}{\partial \tau}+\frac{\partial \psi^{\dagger}}{\partial \xi} \sigma_{3} \frac{\partial \psi}{\partial \xi}+\frac{\partial \psi^{\dagger}}{\partial x} \sigma_{3} \frac{\partial \psi}{\partial x}+\frac{\partial \psi^{\dagger}}{\partial y} \sigma_{3} \frac{\partial \psi}{\partial y}+m^{2} \psi^{\dagger} \sigma_{3} \psi\right\} \xi d \xi
$$

the analogous $\phi$-system Hamiltonian $H^{\phi}$, and the interaction Hamiltonian $H_{\text {int }}$, which is given by Eq.(6.2). Like the total achronal spin, $H^{\text {total }}$ is also conserved throughout the interaction process.

Besides the four conserved observables, Eqs.(6.10) and (5.1), there is also the conserved "total charge" operator Q

$$
Q=\langle\psi, \psi\rangle+\langle\phi, \phi\rangle \quad \text { (total charge) }
$$

for the two interacting Klein Gordon systems.

\section{Spectral Decomposition}

For a multiparticle system the spectral decomposition of an observable invariably highlights physical properties which would otherwise go unnoticed. The achronal spin, boost energy and the charge of a free Klein-Gordon system are no exceptions. Consider the observables $\overrightarrow{J^{\psi}}, H^{\psi}$ and charge $Q$ for a free $\psi$-system. Taking note that its Heisenberg operator is given by Eq.(5.18), one easily finds, with the help of Eqs.(5.9) and (6.11a), that the spectral decompositions of Eq.(6.5), (6.12) and (6.13) are

$$
\begin{aligned}
J_{1}^{\psi} & =\frac{1}{2} \int_{-\infty}^{\infty}\left\{\left(\tilde{a}_{\omega}^{*} \tilde{b}_{\omega}^{*}+\tilde{a}_{\omega} \tilde{b}_{\omega}\right) \frac{\cosh \pi \omega}{\sinh \pi \omega}+\left(\tilde{a}_{\omega} \tilde{a}_{\omega}^{*}+\tilde{b}_{\omega}^{*} \tilde{b}_{\omega}\right) \frac{1}{\sinh \pi \omega}\right\} d \omega \\
J_{2}^{\psi} & =\frac{i}{2} \int_{-\infty}^{\infty}\left(\tilde{a}_{\omega} \tilde{b}_{\omega}-\tilde{a}_{\omega}^{*} \tilde{b}_{\omega}^{*}\right) d \omega \\
J_{3}^{\psi} & =\frac{1}{2} \int_{-\infty}^{\infty}\left\{\left(\tilde{a}_{\omega}^{*} \tilde{b}_{\omega}^{*}+\tilde{a}_{\omega} \tilde{b}_{\omega}\right) \frac{1}{\sinh \pi \omega}+\left(\tilde{a}_{\omega} \tilde{a}_{\omega}^{*}+\tilde{b}_{\omega}^{*} \tilde{b}_{\omega}\right) \frac{\cosh \pi \omega}{\sinh \pi \omega}\right\} d \omega \\
H^{\psi} & =\int_{-\infty}^{\infty} \omega\left(\tilde{a}_{\omega} \tilde{a}_{\omega}^{*}-\tilde{b}_{\omega}^{*} \tilde{b}_{\omega}\right) d \omega \\
Q & =\frac{1}{2} \int_{-\infty}^{\infty}\left(\tilde{a}_{\omega}^{*} \tilde{a}_{\omega}-\tilde{b}_{\omega} \tilde{b}_{\omega}^{*}\right) d \omega .
\end{aligned}
$$

One readily verifies the familiar $S U(1,1)$ commutation relations

and also

$$
\begin{aligned}
& {\left[J_{1}^{\psi}, J_{2}^{\psi}\right]=-i J_{3}^{\psi}} \\
& {\left[J_{2}^{\psi}, J_{3}^{\psi}\right]=i J_{1}^{\psi}} \\
& {\left[J_{3}^{\psi}, J_{1}^{\psi}\right]=i J_{2}^{\psi}}
\end{aligned}
$$




$$
\begin{aligned}
{\left[H^{\psi}, \overrightarrow{J^{\psi}}\right] } & =0 \\
{\left[Q, \overrightarrow{J^{\psi}}\right] } & =0
\end{aligned}
$$

Thus, as expected, achronal spin is invariant under boost.

Note that an equivalent set of achronal spin operators would have been

$$
\begin{aligned}
J_{1}^{\prime} \psi & =\frac{1}{2} \int_{-\infty}^{\infty}\left(\tilde{a}_{\omega}^{*} \tilde{b}_{\omega}^{*}+\tilde{a}_{\omega} \tilde{b}_{\omega}\right) d \omega \\
J_{2}^{\prime} \psi & =\frac{i}{2} \int_{-\infty}^{\infty}\left(\tilde{a}_{\omega} \tilde{b}_{\omega}-\tilde{a}_{\omega}^{*} \tilde{b}_{\omega}^{*}\right) d \omega \\
J_{3}^{\prime} \psi & =\frac{1}{2} \int_{-\infty}^{\infty}\left(\tilde{a}_{\omega} \tilde{a}_{\omega}^{*}+\tilde{b}_{\omega}^{*} \tilde{b}_{\omega}\right) d \omega .
\end{aligned}
$$

Both the original $J_{i}^{\psi}$ 's and the newly defined $J_{i}^{\prime} \psi$ 's obey the same commutation relations. If one pictures the spectral components of $J_{1}^{\prime} \psi, J_{2}^{\prime} \psi$, and $J_{3}^{\prime} \psi$ as three orthogonal basis vectors in a three dimensional Lorentz space, then the spectral components of $J_{1}^{\psi}, J_{2}^{\psi}$, and $J_{3}^{\psi}$ form an alternate Lorentz-orthogonal basis with $J_{3}^{\psi}$ pointing along the direction of the Planckian vector, Eq.(5.12). The Lorentz rotation which relates the two sets of basis vectors was induced by the spinor transformation which relates Eq.(5.15) to Eq.(5.18).

\section{Why Achronal Spin?}

To answer this question let us first compare (i) a system of cavity modes in thermalequilibrium and (ii) a single-charge quantum system viewed relative to spacetime-translated paired accelerated frames.

Both systems carry the Planckian power and fluctuation spectra as their characteristic signature. However considerably more important is their difference. In one case these spectra express, via the moments of a thermal density matrix, the statistical equilibrium of the infinity system. In the second case, they express,via the expectation values relative to pure quantum states, the spacetime translation invariance of the single-charge system.

What does this mixed state versus pure state distinction signify? For the cavity mode system the significance is well known: the two spectra, Planckian and fluctuation, imply the existence of photons within the statistical thermodynamic setting. This we have learned from Planck and Einstein.

Is there a corresponding significance for the single-charge system? Let us assume that the spectra of quantum mechanical expectation values given by Eq.(5.22) or (5.23) lend themselves to being measured by an experiment. If carried out, such an experiment would imply the existence of achronal spin, a quantum mechanical expression of the acceleration-induced partitioning of spacetime into pairs of adjacent but causally disconnected (i.e. "achronally" related) Rindler sectors. 
Achronal spin is not limited to single-charge quantum systems. Its extension to infinite (e.g. multimode) quantum systems leads to the achronal spin densitycurrent, which satisfies a differential conservation law. As a consequence total achronal spin is neither created nor destroyed. This makes achronal spin a macroscopic property of matter.

The second reason for achronal spin is geometrical. Different spacetime frameworks demand different ways of characterizing the dynamics of physical systems, for example, the two interacting quantum systems described in Section 6 .

(1) Relative to an inertial frame use energy, momentum, and charge.

(2) Relative to a binary frame use achronal spin, boost energy, and charge.

Thus the use of energy-momentum as a property of matter is predicated on locally translation invariant inertial ("free fall") frames. In other words, to each event of spacetime there is attached what mathematicians call a tangent space. By contrast, the use of "boost energy" and achronal spin as a property of matter is predicated on paired accelerated frames, one paired frame attached to each event of spacetime.

The geometrical difference, inertial frames vs. paired accelerated frames, goes hand in hand with with the physical difference, energy and momentum vs. achronal spin and boost energy.

Which do we choose, and why? The choice is between two geometrical views of flat Minkowski spacetime. Its invariance group is the Poincare group, the (semi) direct product of translations and Lorentz rotations around an event.

The first choice is implemented by trivializing translation invariance, i.e. by expressing quantum mechanical processes in terms of inertial, i.e. translation eigenfunctions ("plane waves"). This is done in all the text books. Lorentz invariance is imposed as an afterthought on the tangent space at each event. This imposition is done by endowing that tangent space with a Lorentz metric and by requiring Lorentz invariance, i.e. that all physical(ly measurable) properties be expressible in terms of the scalars, vectors, spinors etc. under Lorentz transformations.

The second choice is implemented by trivializing Lorentz invariance, i.e. by expressing quantum mechanical processes in terms of boost eigen functions (for a 2-D Rindler frame) or their generalization (for a 4-D spherical Rindler frame as in Figure 1). Physical properties in different pairs of accelerated frames are then related by the appropriate translation transformation.

In brief, the difference between the two choices consists of interchanging the role that the translation group and the Lorentz play in physical processes.

The choice of groups is, however, not merely a matter of taste. In a pair of accelerated frames the boost eigenstate description of a particle implies a qualitatively new feature, the particle's achronal spin. An inertial frame with its translation eigenstates can not make any such claim.

\section{References}

[1] P. C. W. Davies, J. Phys. A: Math. Gen. 8, 365 (1975). 
[2] W. G. Unruh, Phys. Rev. D 15, 870 (1976).

[3] P. Candelas and D. Deutsch, Proc. R. Soc. London A354, 79 (1977)

[4] P. Candelas and D. Deutsch, Proc. R. Soc. London A362, 251 (1978)

[5] M. Soffel, B. Mueller, W. Greiner, Phys. Rev. D22, 1935 (1980)

[6] W. G. Unruh and N. Weiss, Phys. Rev. D29, 1656 (1984)

[7] J. A. Wheeler, A Journey into Gravity and Spacetime (Scientific American Library, New York, 1990)

[8] C.W. Misner, K.S. Thorne, and J.A. Wheeler, Gravitation (W.H. Freeman and Co., San Francisco, 1973) ch. 15

[9] U.H. Gerlach, Phys. Rev. D28, 761 (1983)

[10] The $\theta, \phi$, and $\tau$ coordinates are what in Euclidean space correspond to the Euler parameters on a three-sphere. They are not to be confused with the $\theta, \phi$, and $\tau$ coordinates of Eq.(4.1), which are what in Euclidean space corresponds to the usual spherical coordinates of a three-sphere. The coordinate transformation Eq.(4.4) is a lower dimensional adoptation of the one Rindler [W. Rindler, Found. of Physics 15, 545 (1985)] used to identify the world line dependent event horizons on the De Sitter hyperboloid.

[11] C.W. Misner, K.S. Thorne, and J.A. Wheeler, Gravitation (W.H. Freeman and Co., San Francisco, 1973) p880

[12] Obtained by inverting Eqs.(4.2) in U.H. Gerlach, Phys.Rev. D 38, 514 (1988), or in gr-qc/9910097.

[13] Integration over $x$ and $y$ is understood, but suppressed throughout this article.

[14] Reference to the modes $\exp \left(i k_{x} x\right)$ and $\exp \left(i k_{y} y\right)$ as well as the corresponding mode integrations are understood, but suppressed throughout this article.

[15] The arguement leading to the angle of inclination, Eq.(5.24), from the precession axis (5.22) holds only for a superposition of two plane wave states. It does not hold for a general superposition of plain wave states, as claimed in the original version of this article published in the MG7 Proceedings.

[16] B. DeWitt in General Relativity: An Einstein centenary survey, edited by S.W. Hawking and W. Israel (Cambridge University Press, New York, 1979) Ch. 14.2 .2

[17] N.D. Birrell and P.C.W. Davies, Quantum fields in curved space (Cambridge University Press, New York, 1984) Ch. 3.3

[18] W.G. Unruh and R.M. Wald, Phys. Rev. D29, 1047 (1984)

[19] D.J. Raine, D.W. Sciama, and P.G. Grove, Proc. Roy. Soc.A435, 205 (1991); P.G. Grove, Class. Quantum Grav. 3, 801 (1986)

[20] W.G. Unruh, Phys. Rev. D46, 3271 (1992) 